International Journal of Pure and Applied Mathematics

Volume 86 No. 4 2013, 607-619

ISSN: 1311-8080 (printed version); ISSN: 1314-3395 (on-line version)

url: http://www.ijpam.eu

doi: http://dx.doi.org/10.12732/ijpam.v86i4.2

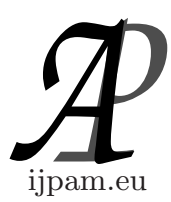

\title{
REGULAR WEAKLY CLOSED SETS IN IDEAL TOPOLOGICAL SPACES
}

\author{
A. Vadivel $^{1} \S$, Mohanarao Navuluri ${ }^{2}$ \\ ${ }^{1,2}$ Department of Mathematics (FEAT) \\ Annamalai University \\ Annamalainagar, Tamil Nadu, 608 002, INDIA
}

\begin{abstract}
In this paper, we define $I_{r w}$-closed sets and $I_{r w}$-open sets, characterize these sets and discuss their properties. Then, we define $\vee_{r s}$-sets and $\wedge_{r s}$-sets and discuss the relation between them. Also, we give characterizations of $I_{r w}$-closed sets and $r w$-closed sets.
\end{abstract}

A separation axiom stronger than $r s T_{I^{-}}$space is defined and various characterizations are given.

AMS Subject Classification: 54A05, 54A10, 54D15

Key Words: $\quad I_{r w^{-c l o s e d}} I_{r w^{-o p e n}}, \mathcal{N}_{I^{-}} r w$-set, $\wp_{I^{-}} r w$-set, $\vee_{r s^{-}}$-set, $\wedge_{r s^{-}}$-set, $I . \vee_{r s}$-set, $I . \wedge_{r s}$-set, $r s T_{I^{-}}$spaces

\section{Introduction}

An ideal $I$ on a topological space $(X, \tau)$ is a nonempty collection of subsets of $X$ which satisfies (i) $A \in I$ and $B \subseteq A$ implies $B \in I$ and (ii) $A \in I$ and $B \in I$ implies $A \cup B \in I$. Given a topological space $(X, \tau)$ with an ideal $I$ on $X$ and if $\wp(X)$ is the set of all subsets of $X$, a set operator $(.)^{*}: \wp(X) \rightarrow$ $\wp(X)$, called a local function [6] of $A$ with respect to $\tau$ and $I$ is defined as follows: for $A \subseteq X, A^{*}(I, \tau)=\{x \in X \mid U \cap A \notin I$ for every $U \in \tau(x)\}$ where $\tau(x)=\{U \in \tau \mid x \in U\}$. We will make use of the basic facts about the local

Received: December 10, 2012

(C) 2013 Academic Publications, Ltd.

${ }^{\S}$ Correspondence author url: www.acadpubl.eu 
function [[5], Theorem 2.3] without mentioning it explicitly. In particular, the local function is monotonic. A Kuratowski closure operator $c l^{*}($.$) for a topology$ $\tau^{*}(I, \tau)$, called the $*$ - topology, finer than $\tau$ is defined by $c l^{*}(A)=A \cup A^{*}(I, \tau)$ [16]. Clearly, if $I=\{\phi\}$, then $\operatorname{cl}^{*}(A)=\operatorname{cl}(A)$ for every subset $A$ of $X$. When there is no chance for confusion, we will simply write $A^{*}$ for $A^{*}(I, \tau)$ and $\tau^{*}$ for $\tau^{*}(I, \tau)$. If $I$ is an ideal on $X$, then $(X, \tau, I)$ is called an ideal space. A subset $A$ of an ideal space $(X, \tau, I)$ is $*$-closed $\left(\tau^{*}\right.$-closed) [5] (resp. *-dense in itself [4]) if $A^{*} \subseteq A$ (resp. $A \subseteq A^{*}$ ). A subset $A$ of an ideal space $(X, \tau, I)$ is $I_{g}$-closed [3] if $A^{*} \subseteq U$ whenever $U$ is open and $A \subseteq U$. By Example 2.1(b) of [11], every $*$-closed and hence every closed set is $I_{g}$-closed. A subset $A$ of an ideal space $(X, \tau, I)$ is said to be $I_{g}$-open if $X-A$ is $I_{g}$-closed. An ideal space $(X, \tau, I)$ is said to be a $T_{I}$-space [3] if every $I_{g}$-closed set is $*$-closed. An ideal $I$ is said to be codense [5] or a boundary ideal [16] if $\tau \cap I=\{\phi\}$. $\mathcal{N}$ denotes the ideal of all nowhere dense subset in $(X, \tau)$. In this paper, we define $I_{r w^{-}}$-closed and $I_{r w^{-}}$open sets in ideal spaces, discuss their properties and give various characterizations. Again, for the collection of these sets, we define $\vee_{r s}$-sets and $\wedge_{r s}$-sets and discuss their properties.

By a space $(X, \tau)$, we always mean a topological space $(X, \tau)$ with no separation properties assumed. If $A \subseteq X, \operatorname{cl}(A)$ and $\operatorname{int}(A)$ will, respectively, denote the closure and interior of $A$ in $(X, \tau)$ and $i n t^{*}(A)$ will denote the interior of $A$ in $\left(X, \tau^{*}\right)$. A subset $A$ of a space $(X, \tau)$ is said to be regular open [15] if $A=\operatorname{int}(\operatorname{cl}(A))$ and $A$ is said to be regular closed [15] if $A=\operatorname{cl}(\operatorname{int}(A))$. A subset $A$ of a space $(X, \tau)$ is said to be semi open [7] (resp. $\alpha$-open [12]) if $A \subseteq \operatorname{cl}(\operatorname{int}(A))$ (resp. $A \subseteq \operatorname{int}(\operatorname{cl}(\operatorname{int}(A))))$. The family of all $\alpha$-open sets in a space $(X, \tau)$, denoted by $\tau^{\alpha}$, is a topology on $X$ finer than $\tau$. The closure of a subset $A$ in $\left(X, \tau^{\alpha}\right)$ is denoted by $c l_{\alpha}(A)$. A subset $A$ of a space $(X, \tau)$ is said to be $g$-closed [8] (resp. $\alpha g$-closed [9]) if $c l(A) \subseteq U\left(\operatorname{resp} . \quad l_{\alpha}(A) \subseteq U\right)$ whenever $A \subseteq U$ and $U$ is open. A subset $A$ of a space $(X, \tau)$ is said to be regular semiopen [2] if there is a regular open set $U$ such that $U \subseteq A \subseteq \operatorname{cl}(U)$. A subset $A$ of a space $(X, \tau)$ is said to be $r w$-closed [1] if $\operatorname{cl}(A) \subseteq U$ whenever $A \subseteq U$ and $U$ is regular semiopen. $A$ is said to be $r w$-open (resp. $g$-open) if $X-A$ is $r w$-closed (resp. $g$-closed). The following lemmas will be useful in the sequel.

Lemma 1.1.. (see [14], Lemma 1.2) Let $(X, \tau, I)$ be an ideal space and $A \subseteq X$. If $A \subseteq A^{*}$, then $A^{*}=\operatorname{cl}\left(A^{*}\right)=\operatorname{cl}(A)=c l^{*}(A)$.

Lemma 1.2.. (see [14], Lemma 1.1) Let $(X, \tau, I)$ be an ideal space. Then $I$ is codense if and only if $A \subseteq A^{*}$ for every semiopen set $A$ in $X$. 


\section{2. $I_{r w^{-C l o s e d}}$ and $I_{r w^{-}}$-Open Sets}

A subset $A$ of an ideal space $(X, \tau, I)$ is said to be a regular weakly closed set with respect to the ideal $I$ ( $I_{r w^{-}}$-losed) if $A^{*} \subseteq U$ whenever $A \subseteq U$ and $U$ is regular semiopen. $A$ is called a regular weakly open set ( $I_{r w}$-open) if $X-A$ is an $I_{r w^{-}}$-closed set. Clearly, every $I_{g}$-closed set is $I_{r w}$-closed and every $r w$-closed set is an $I_{r w}$-closed set. The following Example 2.1(a) shows that an $I_{r w}$-closed set is not an $I_{g}$-closed set and Example 2.1(b) shows that an $I_{r w}$-closed set is not an $r w$-closed set. Theorem 2.2. shows that $I_{r w}$-closed sets is finitely additive and Example 2.3 shows that the finite intersection of $I_{r w}$-closed sets is not an $I_{r w^{-}}$-closed set. If $I=\{\phi\}$, then $\operatorname{cl}(A)=c l^{*}(A)=A^{*}$ for every subset $A$ of $X$ and so $I_{r w}$-closed sets coincide with $r w$-closed sets.

Example 2.1. (a) Let $X=\{a, b, c\}, \tau=\{\phi,\{a\},\{c\},\{a, c\}, X\}$ and $I=\{\phi,\{c\}\}$. Then $A=\{a, c\}$ is not regular semiopen. So the only regular semiopen set containing $A$ is $X$ and hence $A$ is $I_{r w}$-closed. Since $A$ is open and $A^{*}=\{a, b\} \nsubseteq A, A$ is not an $I_{g}$-closed set.

(b) Let $X$ and $\tau$ be as in Example 2.1 (a). Let $I=\wp(X)$. Since every subset of $X$ is $*$-closed, every subset of $X$ is $I_{r w}$-closed. Since $\{a\}$ is regular semiopen and not closed, $\{a\}$ is not rw-closed.

Theorem 2.1.. Every rw-closed set is $I_{r w}$-closed.

Proof. Let $A$ be any $r w$-closed set in $(X, \tau, I)$. Then $\operatorname{cl}(A) \subseteq U$ whenever $U$ is regular semiopen and $A \subseteq U$. Since $A^{*} \subseteq \operatorname{cl}(A) \subseteq U$, we have $A^{*} \subseteq U$ and hence $A$ is $I_{r w}$-closed.

Example 2.2. Let $X=\{a, b, c, d, e\}, \tau=\{\phi,\{a\},\{b\},\{a, b\},\{c, d\}$, $\{a, c, d\},\{b, c, d\},\{a, b, c, d\},\{b, c, d, e\}, X\}$ and $I=\{\phi,\{b\},\{d\},\{b, d\}\}$. Then the set $\{b\}$ is $I_{r w}$-closed but not $r w$-closed.

Remark 2.1.. For a subsets of an ideal space, the following implications hold.

$*$-closed $\Rightarrow I_{g}$-closed $\Rightarrow I_{r w}$-closed $\Leftarrow r w$-closed

when none of the implications is reversible as shown in [[13], Example 3.7]

Theorem 2.2.. In an ideal space $(X, \tau, I)$, the union of two $I_{r w}$-closed sets is an $I_{r w}$-closed set.

Proof. Let $A$ and $B$ be $I_{r w}$-closed sets in $(X, \tau, I)$. Suppose $A \cup B \subseteq U$ and $U$ is regular semiopen. Then $A \subseteq U$ and $B \subseteq U$. By hypothesis, $c l^{*}(A) \subseteq U$ and $c l^{*}(B) \subseteq U$. Therefore, $c l^{*}(A \cup B)=c l^{*}(A) \cup c l^{*}(B) \subseteq U$ which implies that $A \cup B$ is $I_{r w}$-closed. 
Example 2.3. Let $X=\{a, b, c\}, \tau=\{\phi,\{a\},\{c\},\{a, c\}, X\}$ and $I=$ $\{\phi,\{c\}\}$. Let $A=\{a, c\}$ and $B=\{a, b\}$. Since $X$ is the only regular semiopen set containing $A$ and $B, A$ and $B$ are $I_{r w}$-closed. Now $A \cap B=\{a\}$ is regular semiopen and $(A \cap B)^{*}=\{a, b\} \nsubseteq\{a\}$. Therefore, $A \cap B$ is not an $I_{r w}$-closed set.

The following Theorem 2.3. gives a property of $I_{r w}$-closed sets and Example 2.4 shows that the converse of Theorem 2.3. is not true.

Theorem 2.3.. Let $(X, \tau, I)$ be an ideal space and $A \subseteq X$. If $A$ is $I_{r w}$-closed, then $c l^{*}(A)-A$ contains no nonempty regular semiclosed set.

Proof. Let $A$ be an $I_{r w}$-closed set of $(X, \tau, I)$. Suppose a regular semiclosed set $F$ is contained in $c l^{*}(A)-A=c l^{*}(A) \cap(X-A)$. Since $F \subseteq X-A$, we have $A \subseteq X-F$ and $X-F$ is regular semiopen. Therefore, $c l^{*}(A) \subseteq X-F$ and so $F \subseteq X-c l^{*}(A)$. Already, we have $F \subseteq c l^{*}(A)$ and so $F=\phi$. Hence $c l^{*}(A)-A$ contains no nonempty regular semiclosed sets.

Example 2.4. Let $X=\{a, b, c, d\}, \tau=\{\phi,\{a\},\{b\},\{a, b\},\{a, b, c\},\{a, b, d\}$, $X\}$ and $I=\{\phi\}$. Let $A=\{a\}$. Then $c l^{*}(A)-A=c l(A)-A=\{a, c, d\}-\{a\}=$ $\{c, d\}$ which does not contain any nonempty regular semiclosed set. But $A$ is not $I_{r w}$-closed, since $A$ is regular semiopen and $c l^{*}(A)=c l(A)=\{a, c, d\} \nsubseteq \nsubseteq A$.

Theorem 2.4.. If $A$ is an $I_{r w}$-closed subset of an ideal space $(X, \tau, I)$ and $A \subseteq B \subseteq c l^{*}(A)$, then $B$ is also $I_{r w}$-closed. In particular, the *-closure of every $I_{r w}$-closed set is an $I_{r w}$-closed set.

Proof. Suppose $B \subseteq U$ and $U$ is regular semiopen. Since $A$ is $I_{r w}$-closed and $A \subseteq U, c l^{*}(A) \subseteq U$. Since $B \subseteq c l^{*}(A), c l^{*}(B) \subseteq c l^{*}(A)$. Therefore, $c l^{*}(B) \subseteq U$ and so $B$ is $I_{r w^{-}}$-closed.

Theorem 2.5.. If $(X, \tau, I)$ is an ideal space and $A$ is a $*$-dense in itself, $I_{r w}$-closed subset of $X$, then $A$ is a $r w$-closed set.

Proof. If $A$ is a *-dense in itself, $I_{r w}$-closed subset of $X$ and $U$ is any regular semiopen set containing $A$, then $c l^{*}(A) \subseteq U$. Since $A$ is $*$-dense in itself, by Lemma 1.1., $\operatorname{cl}(A) \subseteq U$ and so $A$ is $r w$-closed.

Corollary 2.1.. Let $A$ and $B$ be subsets of an ideal space $(X, \tau, I)$ such that $A \subseteq B \subseteq A^{*}$. If $A$ is $I_{r w^{-c l o s e d}}$, then $A$ and $B$ are $r w$-closed.

Proof. Since $A \subseteq B \subseteq A^{*}, A \subseteq B \subseteq c l^{*}(A)$. Since $A$ is $I_{r w^{-c l o s e d}}$, by Theorem 2.4., $B$ is $I_{r w^{-}}$-closed. Since $A \subseteq B \subseteq A^{*}, B^{*}=A^{*}$. Therefore, $A$ and $B$ are $*$-dense in itself. By Theorem 2.5., $A$ and $B$ are $r w$-closed.

Corollary 2.2.. Let $(X, \tau, I)$ be an ideal space where $I$ is codense. If $A$ 
is a semiopen, $I_{r w}$-closed subset of $X$, then $A$ is rw-closed.

Proof. Since $A$ is a semiopen, by Lemma 1.2., $A \subseteq A^{*}$. So, $A$ is $*$-dense in itself and $I_{r w}$-closed. Therefore, $A$ is $r w$-closed, by Theorem 2.5 .

The following Theorem 2.6. gives another property of $I_{r w}$-closed sets.

Theorem 2.6.. Let $(X, \tau, I)$ be an ideal space and $A$ be an $I_{r w}$-closed set. Then the following are equivalent.

(a) $A$ is a $*$-closed set.

(b) $c l^{*}(A)-A$ is a regular semiclosed set.

(c) $A^{*}-A$ is a regular semiclosed set.

Proof. $(a) \Rightarrow(b)$ If $A$ is $*$-closed, then $c l^{*}(A)-A=\phi$ and so $c l^{*}(A)-A$ is regular semiclosed.

$(b) \Rightarrow(a)$ Suppose $c l^{*}(A)-A$ is regular semiclosed. Since $A$ is $I_{r w}$-closed, by Theorem 2.3., $c l^{*}(A)-A=\phi$ and so $A$ is $*$-closed.

$(b) \Leftrightarrow(c)$. The proof follows from the fact that $c l^{*}(A)-A=A^{*}-A$.

Theorem 2.7.. Let $(X, \tau, I)$ be an ideal space and $A$ be an $I_{r w}$-closed set. Then $A \cup\left(X-A^{*}\right)$ is also an $I_{r w}$-closed set.

Proof. Suppose that $A$ is an $I_{r w}$-closed set. If $U$ is any regular semiopen set such that $A \cup\left(X-A^{*}\right) \subseteq U$, then $X-U \subseteq X-\left(A \cup\left(X-A^{*}\right)\right)=(X-A) \cap A^{*}=$ $A^{*}-A$. Since $X-U$ is regular semiclosed and $A$ is $I_{r w^{-}}$-closed, by Theorem 2.3., it follows that $X-U=\phi$ and so $X=U$. Hence $X$ is the only regular semiopen set containing $A \cup\left(X-A^{*}\right)$ and so $A \cup\left(X-A^{*}\right)$ is $I_{r w^{-c l o s e d}}$

The following Example 2.5 shows that the converse of the above Theorem 2.7. is not true.

Example 2.5. Let $(X, \tau)$ and $I$ be as in Example 2.4. Let $A=\{a\}$. Then $A$ is not $I_{r w^{-}}$-closed. Now, $A \cup\left(X-A^{*}\right)=\{a\} \cup(X-\{a, c, d\})=\{a\} \cup\{b\}=$ $\{a, b\}$. Since the only regular semiopen set containing $A \cup\left(X-A^{*}\right)$ is $X$, $A \cup\left(X-A^{*}\right)$ is $I_{r w^{-}}$-closed.

Theorem 2.8.. Let $(X, \tau, I)$ be an ideal space. Then the following are equivalent.

(a) Every subset of $X$ is $I_{r w}$-closed.

(b) Every regular semiopen set is $*$-closed.

Proof. $(a) \Rightarrow(b)$ Suppose every subset of $X$ is $I_{r w}$-closed. If $U$ is regular semiopen, then by hypothesis, $U$ is $I_{r w^{-}}$-closed and so $U^{*} \subseteq U$. Hence $U$ is *-closed. 
$(b) \Rightarrow(a)$ Suppose every regular semiopen set is $*$-closed. Let $A$ be a subset of $X$. If $U$ is a regular semiopen set such that $A \subseteq U$, then $A^{*} \subseteq U^{*} \subseteq U$ and so $A$ is $I_{r w}$-closed.

The following Theorem 2.9. gives a characterization of $I_{r w}$-open sets.

Theorem 2.9.. Let $(X, \tau, I)$ be an ideal space. A subset $A \subseteq X$ is $I_{r w^{-}}$ open if and only if $F \subseteq i n t^{*}(A)$ whenever $F$ is regular semiclosed and $F \subseteq A$.

Proof. Suppose $A$ is $I_{r w}$-open. Let $F$ be a regular semiclosed set contained in $A$. Then $c l^{*}(X-A) \subseteq X-F$ and so $F \subseteq X-c l^{*}(X-A)=i n t^{*}(A)$. Conversely, suppose $X-A \subseteq U$ and $U$ is regular semiopen. By hypothesis, $X-U \subseteq \operatorname{int}^{*}(A)$ which implies that $c l^{*}(X-A) \subseteq U$. Therefore, $X-A$ is $I_{r w}$-closed and so $A$ is $I_{r w^{-o p e n}}$.

Theorem 2.10.. In an ideal space $(X, \tau, I)$, if $A$ is an $I_{r w}$-open set, then $G=X$ whenever $G$ is regular semiopen and $i n t^{*}(A) \cup(X-A) \subseteq G$.

Proof. Let $A$ be an $I_{r w^{-o p e n}}$ set. Suppose $G$ is a regular semiopen set such that $i n t^{*}(A) \cup(X-A) \subseteq G$. Then $X-G \subseteq\left(X-i n t^{*}(A)\right) \cap A=$ $\left(X-i n t^{*}(A)\right)-(X-A)=c l^{*}(X-A)-(X-A)$. Since $X-A$ is $I_{r w}$-closed, by Theorem 2.3., $X-G=\phi$ and so $G=X$.

The following Example 2.6 shows that the converse of the above Theorem 2.10. is not true.

Example 2.6. Let $(X, \tau)$ and $I$ be as in Example 2.4. The nonempty regular semiopen sets are $\{a\},\{b\},\{a, c\},\{a, d\},\{b, c\},\{b, d\},\{a, c, d\},\{b, c, d\}$ and $X$. Let $A=\{b, c, d\}$. Then $\operatorname{int}^{*}(A) \cup(X-A)=\{b\} \cup\{a\}=\{a, b\}$ and $X$ is the only regular semiopen set containing $\operatorname{int}^{*}(A) \cup(X-A)$. Since $A$ is regular semiclosed and $A \nsubseteq i n t^{*}(A)=\operatorname{int}(A)=\{b\}, A$ is not $I_{r w^{-o p e n}}$.

Theorem 2.11.. If $A$ is an $I_{r w}$-closed set in an ideal space $(X, \tau, I)$, then $c l^{*}(A)-A$ is $I_{r w}$-open.

Proof. Since $A$ is $I_{r w^{-c l o s e d}}$, by Theorem 2.3., $\phi$ is the only regular semiclosed set contained in $c l^{*}(A)-A$ and so by Theorem 2.9., $c l^{*}(A)-A$ is $I_{r w^{-}}$ open.

The following Example 2.7 shows that the converse of the above Theorem 2.11. is not true.

Example 2.7. Consider the ideal space $(X, \tau, I)$ of Example 2.4. Let $A=\{a\}$. Then $c l^{*}(A)-A=\operatorname{cl}(A)-A=\{c, d\}$. Since $\phi$ is the only regular semiclosed set contained in $c l^{*}(A)-A$, by Theorem 2.9., $c l^{*}(A)-A$ is $I_{r w^{-o p e n}}$. But $A$ is not $I_{r w}$-closed.

Theorem 2.12.. Let $(X, \tau, I)$ be an ideal space and $A \subseteq X$. If $A$ is 
$I_{r w^{-o p e n}}$ and $\operatorname{int}^{*}(A) \subseteq B \subseteq A$, then $B$ is $I_{r w^{-}}$-open.

Proof. Since $i n t^{*}(A) \subseteq B \subseteq A$, we have $i n t^{*}(A)=i n t^{*}(B)$. Suppose $F$ is regular semiclosed and $F \subseteq B$, then $F \subseteq A$. Since $A$ is $I_{r w}$-open, by Theorem 2.9., $F \subseteq i n t^{*}(A)=i n t^{*}(B)$. So, again by Theorem 2.9., $B$ is $I_{r w}$-open.

Definition 2.1.. A subset $A$ of an ideal topological space $(X, \tau, I)$ is said to be:

1. a $\mathcal{N}_{I}-r w$-set if $A=U \cap V$, where $U$ is a regular semiopen set and $V$ is a *-perfect set.

2. a $\wp_{I}-r w$-set if $A=U \cap V$, where $U$ is a regular semiopen set and $V$ is a *-closed set.

Theorem 2.13.. A subset $A$ of an ideal topological space $(X, \tau, I)$ is a $\mathcal{N}_{I}$-rw-set and a $I_{r w}$-closed set, then $A$ is a $*$-closed set.

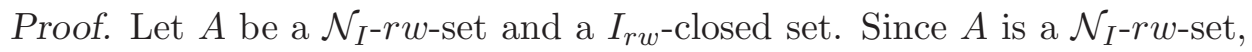
$A=U \cap V$, where $U$ is a regular semiopen set and $V$ is a $*$-perfect set. Now, $A=U \cap V \subseteq U$ and $A$ is a $I_{r w^{-}}$-closed set implies that $A^{*} \subseteq U$. Also, $A=$ $U \cap V \subseteq V$ and $V$ is $*$-perfect set implies that $A^{*} \subseteq V$. Thus, $A^{*} \subseteq U \cap V=A$. Hence, $A$ is a $*$-closed set.

Theorem 2.14.. For a subset $A$ of an ideal topological space $(X, \tau, I)$, the following are equivalent.

1. $A$ is a $*$-closed set.

2. $A$ is a $\wp_{I}-r w$-set and a $I_{r w}$-closed set.

Proof. (1) $\Rightarrow(2)$. Let $A$ be a $*$-closed set and $A=X \cap V$, where $X$ is regular semiopen set and $V$ is a $*$-closed set. Hence, $A$ is a $\wp_{I}$-rw-set. Assume that $A$ be a $*$-closed set and $U$ be a regular semiopen set such that $A \subseteq U$. Then $A^{*} \subseteq U$ and hence $A$ is a $I_{r w^{-}}$closed set.

$(2) \Rightarrow(1)$ Let $A$ be a $\wp_{I}-r w$-set and a $I_{r w^{-}}$-losed set. Since $A$ is a $\wp_{I}$-rw-set, $A=U \cap V$, where $U$ is a regular semiopen set and $V$ is a $*$-closed set. Now, $A \subseteq U$ and $A$ is a $I_{r w^{-}}$-closed set implies that $A^{*} \subseteq U$, Also, $A \subseteq V$ and $V$ is a $*$-closed set implies that $A^{*} \subseteq V$. Thus, $A^{*} \subseteq U \cap V=A$. Hence, $A$ is a *-closed set.

\section{3. $\bigvee_{r s}$ and $\bigwedge_{r s^{-}}$Sets}

Let $(X, \tau)$ be a space. If $B \subseteq X$, we define $B_{r s}^{\vee}=\cup\{F: F \subseteq B$ and $F$ is regular semiclosed $\}$ and $B_{r s}^{\wedge}=\cap\{U: B \subseteq U$ and $U$ is regular semiopen $\}$. The 
following Theorem 3.1. gives properties of these operators, the proof of which is omitted, since the proofs are similar to the corresponding proofs for open sets.

Theorem 3.1.. Let $(X, \tau)$ be a space. If $A$ and $B$ are subsets of $X$, then the following hold.

(a) $\phi_{r s}^{\vee}=\phi$ and $\phi_{r s}^{\wedge}=\phi$.

(b) $X_{r s}^{\vee}=X$ and $X_{r s}^{\wedge}=X$.

(c) $A_{r s}^{\vee} \subseteq A$ and $A \subseteq A_{r s}^{\wedge}$.

(d) $\left(A_{r s}^{\vee}\right)_{r s}^{\vee}=A_{r s}^{\vee}$.

(e) $\left(A_{r s}^{\wedge}\right)_{r s}^{\wedge}=A_{r s}^{\wedge}$.

(f) $A \subseteq B \Rightarrow A_{r s}^{\vee} \subseteq B_{r s}^{\vee}$.

(g) $A \subseteq B \Rightarrow A_{r s}^{\wedge} \subseteq B_{r s}^{\wedge}$.

(h) $A_{r s}^{\vee} \cup B_{r s}^{\vee} \subseteq(A \cup B)_{r s}^{\vee}$.

(i) $A_{r s}^{\wedge} \cup B_{r s}^{\wedge} \subseteq(A \cup B)_{r s}^{\wedge}$.

(j) $(A \cap B)_{r s}^{\vee} \subseteq A_{r s}^{\vee} \cap B_{r s}^{\vee}$.

(k) $(A \cap B)_{r s}^{\wedge} \subseteq A_{r s}^{\wedge} \cap B_{r s}^{\wedge}$.

(l) $A_{r s}^{\vee} \subseteq A^{\vee}$ and $A_{r s}^{\wedge} \supseteq A^{\wedge}$.

A subset $B$ of a space $(X, \tau)$ is said to be a $\vee$-set [10] (resp. $\wedge$-set [10]) if $B=B^{\vee}$ (resp. $B=B^{\wedge}$ ) where $B^{\vee}=\cup\{F \mid F \subseteq B, X-F \in \tau\}$ and $B^{\wedge}=\cap\{U \mid B \subseteq U, U \in \tau\}$. A subset $B$ of a space $(X, \tau)$ is said to be a $\vee_{r s^{-}}$set if $B=B_{r s}^{\vee}$. A subset $B$ of $X$ is said to be a $\wedge_{r s}$-set if $B=B_{r s}^{\wedge}$. Every regular semiclosed set is a $\vee_{r s}$-set and every regular semiopen set is a $\wedge_{r s}$-set. But a $\vee_{r s}$-set need not be a regular semiclosed set and $\wedge_{r s}$-set need not be a regular semiopen set.

Theorem 3.2.. Let $(X, \tau)$ be a space and $A$ be a subset of $X$. Then the following hold.

(a) If $A$ is a $\vee_{r s}$-set, then it is a $\vee$-set.

(b) If $A$ is a $\wedge_{r s}$-set, then it is a $\wedge$-set.

Proof. (a) Always, $A^{\vee} \subseteq A$. Since $A$ is a $\vee_{r s^{-}}$set, $A=A_{r s}^{\vee} \subseteq A^{\vee}$, by Theorem 3.1.(l). Therefore, $A=A^{\vee}$ and so $A$ is a $\vee$-set.

(b) Clearly, $A \subseteq A^{\wedge}$. Since $A$ is a $\wedge_{r s}$-set, $A=A_{r s}^{\wedge} \supseteq A^{\wedge}$ and so $A$ is a $\wedge$-set.

The following Example 3.1 shows that a $\vee$-set need not be a $\vee_{r s}$-set. 
Example 3.1. Consider the space $(X, \tau)$ of Example 2.6 Let $A=\{c, d\}$. Since $A$ is closed, it is a $\vee$-set. But $A_{r s}^{\vee}=\phi$, since there is no regular semiclosed set contained in $A$ and so $A$ is not a $\vee_{r s}$-set.

Theorem 3.3.. Let $(X, \tau)$ be a space. Then $(X-B)_{r s}^{\wedge}=X-B_{r s}^{\vee}$ for every subset $B$ of $X$.

Proof. The proof follows from the definitions.

Corollary 3.1.. Let $(X, \tau)$ be a space. Then $(X-B)_{r s}^{\vee}=X-B_{r s}^{\wedge}$ for every subset $B$ of $X$.

Corollary 3.2.. Let $(X, \tau)$ be a space. Then, a subset $B$ of $X$ is $\vee_{r s}$-set if and only if $X-B$ is a $\wedge_{r s}$-set.

Remark 3.1.. Let $(X, \tau, I)$ be an ideal space. It is clear that a subset $A$ of $X$ is $I_{r w^{-}}$-closed if and only if $c l^{*}(A) \subseteq A_{r s}^{\wedge}$.

Corollary 3.3.. Let $A$ be a $\wedge_{r s}$-set in $(X, \tau, I)$. Then $A$ is $I_{r w}$-closed if and only if $A$ is $*$-closed.

If $I=\{\phi\}$, in Remark 3.1. and Corollary 3.3., we get the following Corollary 3.4. which gives characterizations of $r w$-closed sets.

Corollary 3.4.. Let $(X, \tau)$ be a space and $A \subseteq X$. Then the following hold.

(a) $A$ is rw-closed if and only if $\operatorname{cl}(A) \subseteq A_{r s}^{\wedge}$.

(b) If $A$ is a $\wedge_{r s}$-set, then $A$ is rw-closed if and only if $A$ is closed.

Theorem 3.4.. Let $(X, \tau, I)$ be an ideal space and $A \subseteq X$. If $A_{r s}^{\wedge}$ is $I_{r w}$-closed, then $A$ is also $I_{r w}$-closed.

Proof. Suppose that $A_{r s}^{\wedge}$ is $I_{r w}$-closed. If $A \subseteq U$ such that $U$ is regular semiopen, then $A_{r s}^{\wedge} \subseteq U$. Since $A_{r s}^{\wedge}$ is $I_{r w}$-closed, $c l^{*}\left(A_{r s}^{\wedge}\right) \subseteq U$. Since $A \subseteq A_{r s}^{\wedge}$, it follows that $c l^{*}(A) \subseteq U$ and so $A$ is $I_{r w^{-}}$-closed.

The following Example 3.2 shows that the converse of Theorem 3.4. is not true.

Example 3.2. Let $X=\{a, b, c, d, e\}, \tau=\{\phi,\{c\},\{e\},\{c, e\},\{a, b, c\}$, $\{c, d, e\},\{a, b, c, e\}, X\}$ and $I=\{\phi\} . A=\{a, b\}$ is $*$-closed and hence $I_{r w^{-}}$ closed. $A_{r s}^{\wedge}=\{a, b, c\}$ is regular semiopen but it is not $*$-closed. Therefore, $A_{r s}^{\wedge}$ is not an $I_{r w}$-closed set.

In an ideal space $(X, \tau, I)$, a subset $B$ of $X$ is said to be an $I . \wedge_{r s}$-set if $B_{r s}^{\wedge} \subseteq F$ whenever $B \subseteq F$ and $F$ is $*$-closed. A subset $B$ of $X$ is called $I . \vee_{r s^{-}}$ set if $X-B$ is an $I . \wedge_{r s}$-set. Every $\vee_{r s}$-set is an $I . \vee_{r s}$-set and every $\wedge_{r s}$-set is an 
$I . \wedge_{r s}$-set. The following Example 3.3 shows that an $I . \wedge_{r s}$-set is not a $\wedge_{r s}$-set. Theorem 3.5. below gives a characterization of $I . \vee_{r s}$-sets.

Example 3.3. Let $(X, \tau)$ and $I$ be as in Example 2.4. Let $A=\{a, b, c\}$. Then the only $*$-closed set containing $A$ is $X$ and so $A$ is an $I . \wedge_{r s}$-set. Since $A_{r s}^{\wedge}=X, A$ is not a $\wedge_{r s}{ }^{- \text {set. }}$.

Theorem 3.5.. A subset $A$ of an ideal space $(X, \tau, I)$ is an $I . \vee_{r s}$-set if and only if $U \subseteq A_{r s}^{\vee}$ whenever $U \subseteq A$ and $U$ is *-open.

Proof. The proof follows from the definitions and Theorem 3.3..

Theorem 3.6.. Let $(X, \tau, I)$ be an ideal space. Then for each $x \in X,\{x\}$ is either *-open or an $I . \vee_{r s}$-set.

Proof. Suppose $\{x\}$ is not $*$-open for some $x \in X$. Then $X-\{x\}$ is not $*-$ closed and so the only $*$-closed set containing $X-\{x\}$ is $X$. Therefore, $X-\{x\}$ is an $I . \wedge_{r s}$-set and hence $\{x\}$ is an $I . \vee_{r s}$-set.

Theorem 3.7.. Let $B$ be an $I . \vee_{r s}$-set in $(X, \tau, I)$. Then for every $*$-closed set $F$ such that $B_{r s}^{\vee} \cup(X-B) \subseteq F, F=X$ holds.

Proof. Let $B$ be an $I . \vee_{r s}$-set. Suppose $F$ is a *-closed set such that $B_{r s}^{\vee} \cup$ $(X-B) \subseteq F$. Then $X-F \subseteq X-\left(B_{r s}^{\vee} \cup(X-B)\right)=\left(X-B_{r s}^{\vee}\right) \cap B$. Since $B$ is an $I . \vee_{r s}$-set and the $*$-open set $X-F \subseteq B$, by Theorem 3.5., $X-F \subseteq B_{r s}^{\vee}$. Also, $X-F \subseteq X-B_{r s}^{\vee}$. Therefore, $X-F \subseteq B_{r s}^{\vee} \cap\left(X-B_{r s}^{\vee}\right)=\phi$ and hence $F=X$.

Corollary 3.5.. Let $B$ be an $I . \vee_{r s}$-set in an ideal space $(X, \tau, I)$. Then $B_{r s}^{\vee} \cup(X-B)$ is $*$-closed if and only if $B$ is a $\vee_{r s}$-set.

Proof. Let $B$ be an $I . \vee_{r s}$-set in $(X, \tau, I)$. If $B_{r s}^{\vee} \cup(X-B)$ is $*$-closed, then by Theorem 3.7., $B_{r s}^{\vee} \cup(X-B)=X$ and so $B \subseteq B_{r s}^{\vee}$. Therefore, $B=B_{r s}^{\vee}$ which implies that $B$ is a $\vee_{r s}$-set. Conversely, suppose $B$ is an $\vee_{r s}$-set. Then $B=B_{r s}^{\vee}$ and so $B_{r s}^{\vee} \cup(X-B)=B \cup(X-B)=X$ is $*$-closed.

Theorem 3.8.. Let $B$ be a subset of an ideal space $(X, \tau, I)$ such that $B_{r s}^{\vee}$ is *-closed. If $X$ is the only *-closed set containing $B_{r s}^{\vee} \cup(X-B)$, then $B$ is an $I . \vee_{r s}$-set.

Proof. Let $U$ be a *-open set contained in $B$. Since $B_{r s}^{\vee}$ is *-closed, $B_{r s}^{\vee} \cup$ $(X-U)$ is $*$-closed. Also, $B_{r s}^{\vee} \cup(X-B) \subseteq B_{r s}^{\vee} \cup(X-U)$. By hypothesis, $B_{r s}^{\vee} \cup(X-U)=X$. Therefore, $U \subseteq B_{r s}^{\vee}$ which implies by Theorem 3.5., that $B$ is an $I . \vee_{r s}$-set.

An ideal space $(X, \tau, I)$ is said to be an $r s T_{I}$-space if every $I_{r w}$-closed set is a $*$-closed set. Clearly, every $r s T_{I^{-}}$-space is a $T_{I}$-space but the converse is not 
true, since an $I_{r w}$-closed set need not be an $I_{g}$-closed set by Example 2.1(a). The following Theorem 3.9. gives characterizations of $r s T_{I}$-spaces.

Theorem 3.9.. In an ideal space $(X, \tau, I)$, the following statements are equivalent.

(a) $(X, \tau, I)$ is an $r s T_{I}$-space.

(b) Every $I . \vee_{r s}$-set is a $\vee_{r s}$-set.

(c) Every $I . \wedge_{r s}$-set is a $\wedge_{r s}$-set.

Proof. $(a) \Rightarrow(b)$ If $B$ is an $I . \vee_{r s}$-set which is not a $\vee_{r s}$-set, then $B_{r s}^{\vee} \varsubsetneqq B$. So, there exists an element $x \in B$ such that $x \notin B_{r s}^{\vee}$. Then $\{x\}$ is not regular semiclosed. Therefore, $X-\{x\}$ is not regular semiopen and so it follows that $X-\{x\}$ is $I_{r w}$-closed. By hypothesis, $X-\{x\}$ is $*$-closed. Since $x \in B$ and $x \notin B_{r s}^{\vee}, B_{r s}^{\vee} \cup(X-B) \subseteq X-\{x\}$. Since $X-\{x\}$ is $*$-closed, by Theorem 3.7., $X-\{x\}=X$, a contradiction.

(b) $\Rightarrow(a)$ Suppose that there exists an $I_{r w}$-closed set $B$ which is not $*-$ closed. Then, there exists $x \in c l^{*}(B)$ such that $x \notin B$. By Theorem 3.6., $\{x\}$ is either $*$-open or an $I . \vee_{r s}$-set. If $\{x\}$ is $*$-open, then $\{x\} \cap B=\phi$ is a contradiction to the fact that $x \in c l^{*}(B)$. If $\{x\}$ is an $I . \vee_{r s}$-set, then $\{x\}$ is a $\vee_{r s}$-set and hence it follows that $\{x\}$ is regular semiclosed. Since $B \subseteq X-\{x\}, X-\{x\}$ is regular semiopen and $B$ is $I_{r w}$-closed, $c l^{*}(B) \subseteq X-\{x\}$, a contradiction to the fact that $x \in c l^{*}(B)$. Therefore, $(X, \tau, I)$ is an $r s T_{I}$-space.

(b) $\Leftrightarrow(c)$ The proof follows from the definition of an $I . \vee_{r s}$ set and from Corollary 3.2.

Theorem 3.10.. An ideal space $(X, \tau, I)$ is an $r s T_{I}$-space if and only if every singleton set in $X$ is either $*$-open or regular semiclosed.

Proof. If $x \in X$ such that $\{x\}$ is not regular semiclosed, then $X-\{x\}$ is not regular semiopen and so it follows that $X-\{x\}$ is $I_{r w}$-closed. By hypothesis, $X-\{x\}$ is $*$-closed and so $\{x\}$ is $*$-open. Conversely, let $A$ be an $I_{r w^{-}}$-closed set and $x \in c l^{*}(A)$. Consider the following two cases:

Case (i): Suppose $\{x\}$ is regular semiclosed. Since $A$ is $I_{r w}$-closed, by Theorem 2.3., $c l^{*}(A)-A$ does not contain a non-empty regular semiclosed set which implies that $x \notin c l^{*}(A)-A$ and so $x \in A$.

Case (ii): Suppose $\{x\}$ is $*$-open. Then $\{x\} \cap A \neq \phi$ and so $x \in A$.

Thus in both cases $x \in A$. Therefore, $A=c l^{*}(A)$ which implies that $A$ is *-closed. So $(X, \tau, I)$ is an $r s T_{I^{-}}$-space. 


\section{References}

[1] S. S. Benchalli and R. S. Wali, On rw-closed sets in topological spaces, Bulletin of the Malaysian Mathematical Sciences and Society, (2) 30 (2) (2007), 99-110.

[2] D. E. Cameron, Properties of $s$-closed spaces, Proc. Amer. Math. Soc., 72 (1978), 581-586.

[3] J. Dontchev, M. Ganster and T. Noiri, Unified approach of generalized closed sets via topological ideals, Math. Japonica, 1999, 49, 395-401.

[4] E. Hayashi, Topologies defined by local properties, Math. Ann., 1964, 156, 205-215.

[5] D. Jankovic, T. R. Hamlett, New topologies from old via ideals, Amer. Math. Monthly, 1990, 97(4), 295-310.

[6] K. Kuratowski, Topology. Vol. I, Academic Press, New York, 1966.

[7] N. Levine, Semi-open sets and semi-continuity in topological spaces, Amer. Math. Monthly, 1963, 70, 36-41.

[8] N. Levine, Generalized closed sets in topology, Rend. Circ. Mat. Palermo, (2), 1970, 19, 89-96.

[9] H. Maki, R. Devi, K. Balachandran, Associated topologies of generalized $\alpha$-closed sets and $\alpha$-generalized closed sets, Mem. Fac. Sci. Kochi Univ. Math., 1994, 15, 51-63.

[10] M. Mrsevic, On pairwise $R_{0}$ and pairwise $R_{1}$ bitopological spaces, Bull. Math. Soc. Sci. Math. R. S. Roumanie, 1986, 30, 141-148.

[11] M. Navaneethakrishnan, J. Paulraj Joseph. g-closed sets in ideal topological spaces, Acta Math. Hungar., 2008, 119(4), 365-371.

[12] O. Njastad, On some classes of nearly open sets, Pacific J. Math., 1965, 15(3), 961-970.

[13] T. Noiri, K. Viswanathan, M. Rajamani, S. Krishnaprakash, On $\omega$-closed sets in ideal topological spaces. (Submitted).

[14] V. Renukadevi, D. Sivaraj and T. Tamizh Chelvam, Properties of topological ideals and Banach category theorem, Kyungpook Math. J., 2005, 45, 199-209. 
[15] M. H. Stone, Application of the theory of boolean rings to general topology, Trans. Amer. Math. Soc., 41 (1937), 374-481.

[16] R. Vaidyanathaswamy, Set Topology, Chelsea Publishing Company, 1946. 
